\title{
Dietary assessment with the online platform MyFitnessPal: a reliable method?
}

\author{
Charlotte Evenepoel ${ }^{1}$, Egbert Clevers ${ }^{1}$, Lise Deroover $^{1}$, Christophe Matthys $^{2}$ and \\ Kristin Verbeke \\ ${ }^{1}$ Translational Research in Gastrointestinal Disorders, KU Leuven, Leuven, Belgium and \\ ${ }^{2}$ Clinical and Experimental Endocrinology, KU Leuven, Leuven, Belgium
}

\section{Abstract}

A major challenge in dietary assessment is the manual pre-processing of dietary data. Digital food registration via online platforms that are coupled to large food databases can automate this process. The accuracy of such platforms depends on the quality of the associated food database. In this study we validated the database of MyFitnessPal (MFP) versus the Belgian food composition database Nubel.

Fifty participants collected a 4-day dietary record using MFP at 2 time points, T1 and T2. Nutrient intake values extracted from MFP at T1 were used as training set to define a cut-off for each parameter (energy intake (EI), carbohydrates $(\mathrm{CH})$, fat $(\mathrm{F})$, protein $(\mathrm{P})$, fibre (Fi), sugar (S), cholesterol (Ch) and sodium (So)) by optimising correlation with Nubel calculated values. Application of these criteria to the values extracted at $\mathrm{T} 2(\mathrm{n}=2826)$ resulted in rejection rate of $2.8 \%$. The remaining values were correlated (Pearson or Spearman) with the Nubel calculated values. Fixed and proportional biases were traced using Bland-Altman analysis and impact on sample size was estimated from a power simulation.

MFP demonstrated strong correlation with the Nubel database for EI $(r=0.95), \mathrm{CH}(\mathrm{r}=0.90), \mathrm{F}(\mathrm{r}=0.90), \mathrm{P}(\mathrm{r}=0.88), \mathrm{Fi}(\mathrm{r}=0.80)$ and $\mathrm{S}(\mathrm{r}=0.77)$, but weak correlations for $\mathrm{Ch}(\rho=0.51)$ and So $(\rho=0.53)$; all $\mathrm{p}<0.001$. Bland-Altman analysis showed no fixed bias between both methods, whereas a proportional bias was found for cholesterol. A practical implication is a loss of statistical power by $5-10 \%$ for EI and macronutrients compared to the Nubel database and the need to increase sample size accordingly.

We conclude that dietary analysis with MFP is accurate and efficient for total energy intake, macronutrients, fibre and sugar, but not for cholesterol and sodium

\section{Conflict of Interest}

"There is no conflict of interest". 